H A R VAR D

\title{
Investment Incentives in Open-Source and Proprietary Two-Sided Platforms
}

Ramon Casadesus-Masanell Gaston Llanes

\section{Working Paper}

12-114

September 6, 2013 


\title{
INVESTMENT INCENTIVES IN OPEN-SOURCE AND PROPRIETARY TWO-SIDED PLATFORMS*
}

\author{
RAMON CASADESUS-MASANELL ${ }^{\dagger}$ AND GASTÓN LLANES ${ }^{\ddagger}$
}

\begin{abstract}
We study incentives to invest in platform quality in open-source and proprietary two-sided platforms. Open platforms have open access, and developers invest to improve the platform. Proprietary platforms have closed access, and investment is done by the platform owner. We present five main results. First, open platforms may benefit from limited developer access. Second, an open platform may lead to higher investment than a proprietary platform. Third, opening one side of a proprietary platform may lower incentives to invest in platform quality. Fourth, the structure of access prices of the proprietary platform depends on (i) how changes in the number of developers affect the incentives to invest in the open platform, and (ii) how investment in the open platform affects the revenues of the proprietary platform. Finally, a proprietary platform may benefit from higher investment in the open platform. This result helps explain why the owner of a proprietary platform such as Microsoft has chosen to contribute to the development of Linux.

KeYwords: Two-Sided Markets, Platform Investment, Network Effects, OpenSource Software, Application Development, Complementarity (JEL: O31, L17, D43).
\end{abstract}

Date: September 6, 2013.

* We thank two anonymous referees and the Editor for helpful comments that have resulted in a much better paper. We also thank Jay Pil Choi, Jacques Crémer, Andrei Hagiu, Hanna Hałaburda, Andrés Hervás-Drane, Elon Kohlberg, Francisco Ruiz-Aliseda, Yaron Yehezkel, David Zvilichovsky, and seminar participants at the 3rd Annual Searle Center Conference on Internet Search and Innovation (Northwestern University), the 39th Annual Conference of the European Association for Research in Industrial Economics (Rome, Italy), the 7th Bi-annual Conference on The Economics of Intellectural Property, Software and the Internet (Toulouse School of Economics), and Pontificia Universidad Católica de Chile. Casadesus-Masanell gratefully acknowledges financial support from the HBS Division of Research. Llanes gratefully acknowledges financial support from FONDECYT Initiation to Research Grant No. 11110043.

$\dagger$ Harvard Business School, casadesus@gmail.com.

¥ Escuela de Administración, Pontificia Universidad Católica de Chile, gaston@llanes.com.ar. 


\section{INTRODUCTION}

While open-source and proprietary platforms have coexisted since the early days of the computing industry, competition between these two modes of development has intensified dramatically following the surge of the Internet in the mid-1990s. Prominent examples include Linux vs. Windows, Open Office vs. Microsoft Office, Firefox vs. Safari, Apache vs. MS Internet Server, and more recently, Google's Android vs. Apple's iOS.

The open-source development model is characterized by two distinctive features: open access (the freedom to use the software free of charge) and open investment (the freedom to modify the source code) 1 Proprietary development, on the other hand, has closed access and closed investment: the platform owner sets access prices and invests centrally to improve its quality. The coexistence of these two diametrically opposed modes of platform governance has sparked a thriving literature on open source examining why individuals and profit-maximizing firms might choose to contribute to open-source development (see Lerner and Tirole, 2005; von Krogh and von Hippel, 2006; Fershtman and Gandal, 2011, for recent surveys).

While insightful and enlightening, theoretical developments on the economics of open source have fallen short of fully embracing the modeling breakthroughs offered by the literature on two-sided platforms of the past decade (e.g., Caillaud and Jullien, 2003; Rochet and Tirole, 2003, 2006; Armstrong, 2006; Hagiu, 2006a; Spulber, 2006; Weyl, 2010). Likewise, while the literature on two-sided platforms has studied some aspects of open platforms, the most distinctive feature of open source (i.e., open investment) has not been considered $!^{2}$

In this paper, we bring together these two streams of work to address the following questions: (i) How are the incentives to invest in platform quality affected by the degree of platform openness? (ii) What is the relation between access and investment strategies? and (iii) How are access prices and incentives to invest in

\footnotetext{
${ }^{1}$ Open access and open investment are complementary but do not always go hand in hand. For example, MS Explorer is an open-access program, but it does not allow for open investment as the source code is not made available to users.

${ }^{2}$ To the literature on two-sided platforms, an open platform is one that offers open access, and a proprietary platform is one that has closed access (the platform sets access prices, positive for at least one side). Thus, this literature is silent about the investment side of platform openness: an open-source platform not only offers open access but it also allows users and developers to invest in platform quality by modifying the source code.
} 
platform quality moderated by competition between open-source and proprietary two-sided platforms?

We set up a model of a platform that brings together users and developers of applications. Users are heterogeneous in their willingness to pay for access to the platform. Developers are heterogeneous in that they bear different costs for developing applications. A proprietary platform chooses how much to invest in platform quality and sets access prices for each side of the market. An open platform may be accessed for free and developers may invest in improving its quality:3 After users and developers have accessed the platform, developers compete to sell applications to users. Users prefer product variety but consider applications as interchangeable. Along with the case of substitute applications whose marginal value decreases with the number of applications available, we study the mirror case of complement applications.

We divide the analysis into two parts. First, we examine models of proprietary and open monopoly platforms; that is, we consider incentives to invest in proprietary and open platforms in isolation from each other and compare equilibrium outcomes. In the second part, we study a mixed-duopoly model with direct competition between both types of platforms.

We obtain the following results. First, open platforms may benefit from limited developer access. The intuition is that the level of investment by developers depends on the expected profit that these investments generate. If the effect of a change in the number of applications on developer revenue decreases with investment in platform quality, a lower number of developers may result in higher investment in the open platform.

Second, if we compare a proprietary and an open platform with the same number of users and developers, the proprietary platform always enjoys more investment. However, an open platform may result in higher investment if it leads to a different number of users or developers. Specifically, the open platform may garner more

\footnotetext{
${ }^{3}$ For concreteness, we focus on developer investment in open platforms and assume away user investment. Recent empirical evidence suggests that a large share of investments in open source are made by firms rather than by users. For example, a 2012 report by the Linux Foundation (Corbet, Kroah-Hartman, and McPherson, 2012) states that seventy-five percent of all Linux kernel development is done by developers who are being paid for their work, and that the top ten organizations sponsoring Linux development are Red Hat, Intel, Novell, IBM, Texas Instruments, Broadcom, Nokia, Samsung, Oracle, and Google. Extending the model to include user innovation would only strengthen our results.
} 
investment only if one of the following conditions is met: (i) the open platform attracts more users, or (ii) the open platform attracts fewer developers and the effect of a change in the number of applications on developer revenue decreases with investment in platform quality, or (iii) the open platform attracts more developers and the effect of a change in the number of applications on developer revenue increases with investment in platform quality.

Third, opening one side of a proprietary platform may lower incentives to invest in platform quality. A closed platform sets access prices to internalize indirect network effects. This allows the platform to capture the entire variation in utility resulting from larger investment. Internalizing network effects is not possible when one of the sides has free access. In this case, the platform captures less of the investment's contribution to consumer utility. As a result, investment incentives are weaker. Lower investment, in turn, may lead to lower levels of adoption by users and developers.

Finally, in a mixed duopoly where a proprietary and an open platform compete to attract single-homing users and where developers can multi-home, we find two results. First, the structure of access prices depends on: (i) how changes in the number of developers affect the incentives to invest in the open platform, and (ii) how investment in the open platform affects the revenues of the proprietary platform. Depending on the sign of these two effects, user and developer access prices may increase or decrease relative to a situation without investment.

We also find that a proprietary platform may benefit from higher investment in the competitor open platform. The intuition is that the number of applications in the open platform is determined by developers' revenues, which increase with platform quality. Under multi-homing, both platforms share applications. A larger number of applications, in turn, may lead to higher revenues for the proprietary platform.

This result explains why proprietary firms may choose to contribute to the development of competing open-source platforms. For example, in a recent report, Corbet, Kroah-Hartman, and McPherson (2012) show that Microsoft ranks 17 in the list of top contributors to Linux. Indeed, while in 2001 Microsoft's CEO Steve Ballmer famously claimed that "Linux is a cancer that attaches itself in an intellectual property sense to everything it touches," in 2010 Jean Paoli (general 
manager of Microsoft's interoperability strategy team) declared: "We love open source."

Our analysis has important managerial implications. First, we show under what conditions open source may lead to high investment in platform quality, which has important implications for profit-maximizing and non-profit firms participating on operating-system or applications markets. Second, we show that access and investment policies have different effects on the equilibrium, but also have important interactions. For example, introducing an open-access policy in an otherwise proprietary platform may improve access incentives, but at the cost of lowering investment incentives. Third, we show how access prices should be set when a proprietary platform competes directly with an open-source platform. Finally, we show that open platforms may be complementary to proprietary platforms, especially when the proprietary platform needs to build an installed base of applications.

The rest of the paper is organized as follows. In the following section, we explain how our paper relates with the extant literature. In Section 2, we present the model. In Section 3, we study and compare monopoly proprietary and open platforms. In Section 4, we study a hybrid model with open access and closed investment. In Section 5, we study a mixed duopoly model in which a proprietary platform and an open platform compete for users. Section 6 concludes. All proofs are in the appendix.

1.1. Related literature. Our paper contributes to the literature on multisided markets and on the economics of open source. A large share of the extant literature on two-sided platforms studies pricing in the presence of network effects (e.g., Spulber, 1996; Caillaud and Jullien, 2003, Rochet and Tirole, 2003, 2006; Armstrong, 2006; Hagiu, 2006a; Nocke, Peitz, and Stahl, 2007; Casadesus-Masanell and Ruiz-Aliseda, 2008; Weyl, 2010). In general terms, the structure of equilibrium prices depends on the relative size of demand elasticities and cross-group externalities, the costs of serving each side of the market, market structure, and whether end-users single-home or multi-home. Although we focus on the incentives to invest in platform quality, we also derive the access prices charged by proprietary platforms in equilibrium and obtain results congruous with the literature. Closer to our setting, Hagiu (2006b) and Economides and Katsamakas 
(2006b) compare proprietary and open platforms. These papers model open platforms as open-access platforms. While we also assume zero access prices for open platforms, we allow for developer innovation to improve platform quality.

Incentives to invest in platform quality in proprietary and open-source two-sided platforms have not been analyzed before. Hagiu (2007), Belleflamme and Peitz (2010), Zhao (2010), and Lin, Li, and Whinston (2011) study sellers' incentives to invest in the quality of the products they sell, rather than on the quality of the platform. Our work is closer to Economides and Katsamakas (2006a) who examine incentives to invest in a one-sided platform with one application developer. These authors compare proprietary and open-source operating systems. In a proprietary operating system, quality-enhancing investments are made by the platform owner; in an open-source operating system, investments are made by the application developer and advanced users. They find that the incentives to invest in the application are generally larger when the platform is open, and that investment in the open-source operating system is larger if there are strong reputation effects from participation in open-source development or a significant part of the users are developers.

Rather than one-sided operating systems, we consider two-sided platforms. In our setting, the proprietary platform chooses access prices for two sides and may subsidize one side in order to better exploit indirect network effects. Moreover, we allow for endogenous platform adoption by users and developers and, contrary to Economides and Katsamakas (2006a), in our model there is always a large number of users and developers. We do not consider the role of reputation from participation in open-source development on developers' incentives to invest. Our analysis thus shows that an open platform may obtain higher investment than a proprietary one even in the absence of reputational concerns.

The early literature on open source was concerned with explaining why individual developers contributed to open-source projects allegedly for free (Lerner and Tirole, 2005; von Krogh and von Hippel, 2006; Fershtman and Gandal, 2011, present excellent surveys). The most common explanations were altruism, personal gratification, peer recognition, and career concerns. We do not consider social preferences or career concerns. Rather, we focus on self-interested agents and examine the value of investments in the platform to the very developers who make those investments. 
Our paper also contributes to an emerging literature in strategy that explores competitive interactions between organizations with different business models. While several formal models of asymmetric competition exist in strategy (mainly, differences in costs, resource endowments, or information), the asymmetries that this literature wrestles with are of a different nature: firms with fundamentally different objective functions, opposed approaches to competing, or different governance structures. Within this literature, papers examining competition between open-source and proprietary software have considered duopoly models of a profit-maximizing, proprietary firm and a community of not-for-profit/nonstrategic open-source developers selling at zero price (Mustonen, 2003; Bitzer, 2004; Gaudeul, 2005; Casadesus-Masanell and Ghemawat, 2006; Economides and Katsamakas, 2006b; Lee and Mendelson, 2008; Casadesus-Masanell and Llanes, 2011). These papers, however, assume that investment incentives are exogenously given (generally, investment in open source is a function of the number of users). The exception is Llanes and de Elejalde (2013), who assume that investment is performed by sellers of complementary goods. In addition, for the most part, the literature on mixed duopoly presents models of one-sided firms. We contribute work in this area by endogenizing developer's investment incentives and by considering interactions between different types of two-sided platforms.

\section{THE MODEL}

We study a two-sided monopoly platform that brings together application developers and users. The platform may be software (e.g., an operating system), hardware (e.g., a DVD player), or a combination of the two (e.g., a video game console). We focus on the incentives to invest in platform quality, that is, on the incentives to develop the software or hardware that constitutes the platform. Although the number of applications is endogenous in our model, we do not study incentives to invest in application quality, which have been studied elsewhere (Hagiu, 2007; Belleflamme and Peitz, 2010; Zhao, 2010; Lin, Li, and Whinston, 2011).

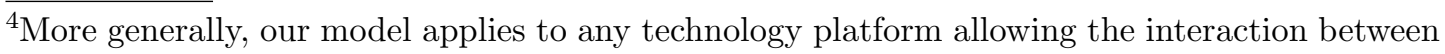
sellers and buyers.
} 
There is a continuum of potential users, $i \in[0, \infty)$, and developers, $j \in[0, \infty) .^{5}$ Users demand applications and run them on the platform. The indirect utility of user $i$ is

$$
u(i)=v(n, x)-\int_{0}^{n} \rho(j) d j-h(i)-p^{u},
$$

where $n$ is the measure of available applications, $x$ is the investment in platform quality, $v(n, x)$ is the gross utility of consuming $n$ applications when the platform has received investment $x, h(i)$ is a user-specific adoption cost, $p^{u}$ is the platform access price for users, and $\rho(j)$ is the price of application $j]^{6}$

We follow the usual convention of representing partial derivatives through subscripts (e.g., $v_{n x}=\frac{\partial^{2} v(n, x)}{\partial n \partial x}$ ), and assume that all functions are three times continuously differentiable.

Users prefer higher quality platforms and application variety, $v_{x}>0$ and $v_{n}>0$. The investment in platform quality and the measure of applications are complements, $v_{n x} \geq 0$. If $v_{n n}=0$, applications are independent in that consuming more of any one application does not affect the marginal utility of consuming any other application. The cases $v_{n n}<0$ and $v_{n n}>0$ correspond to applications being substitutes and complements, respectively. If $v_{n n}<0$, we have $v\left(n_{1}, x\right)+v\left(n_{2}, x\right)>v\left(n_{1}+n_{2}, x\right)$, and applications detract from each other. The reverse is true for complements. Without loss of generality, let $h(0)=0$. Consumers are ordered according to cost so that $h_{i}>0$. Therefore, $h(i) \geq 0$.

Each developer may produce one application. Developer $j$ 's profits are

$$
\pi(j)=\rho(j) m-c(j)-p^{d}-\sigma x(j),
$$

where $m$ is the measure of users, $c(j)$ is a developer-specific development cost, $\sigma$ is the marginal cost of investing in platform quality, $x(j)$ is developer $j$ 's investment in platform quality, and $p^{d}$ is the platform access price for developers. Developers are ordered according to cost so that $c_{j}>0$. Assume $0 \leq c(0) \leq v_{n}(0, x)$, which means that having a positive number of applications is always desirable from a social point of view.

There are two types of platforms. In a proprietary platform, the platform is provided by a profit-maximizing firm, which sets access prices $p^{u}$ and $p^{d}$ and

\footnotetext{
${ }^{5}$ The assumption of a continuum of users and developers is made for easiness of exposition. Our results directly translate to a model with a discrete number of users or developers.

${ }^{6}$ Function $h$ may also be interpreted as a taste-differentiation parameter or transportation cost.
} 
invests in platform quality. Therefore, in this case, developers' investment $x(j)$ is null. In an open-source platform (hereinafter referred to as "open platform"), access to the platform is free, $p^{u}=0$ and $p^{d}=0$, and developers invest in platform quality. Therefore, $x(j)$ may be positive.

As noted in the introduction, the extant literature on open platforms in multisided markets has only considered the zero-price dimension of open source (open access), and has not studied the implications of open source on the incentives for innovation (open investment). We include this important aspect of open platforms in our model and analysis.

\section{MONOPOly PlatFormS}

In this section, we study access and investment incentives in proprietary and open platforms in isolation from each other and compare equilibrium outcomes. We begin by characterizing the socially optimal allocation.

3.1. First best. The social planner chooses $m, n$, and $x$ to maximize the sum of indirect utility and profits:

$$
\begin{aligned}
W & =\int_{0}^{m} u(i) d i+\int_{0}^{n} \pi(j) d j \\
& =m v(n, x)-\int_{0}^{m} h(i) d i-\int_{0}^{n} c(j) d j-\sigma x .
\end{aligned}
$$

For easiness of exposition, in what follows we omit functional arguments when writing equilibrium conditions. Thus, functions $v(n, x)$ and their derivatives are evaluated at the equilibrium values of $m, n$, and $x$. Likewise, functions $h(i)$ and $c(j)$ and their derivatives are evaluated at the marginal user $i=m$ and developer $j=n$.

The equations characterizing the first best (obtained straightforwardly by differentiating $W$ with respect to $m, n$, and $x)$ are

$$
v=h, \quad m v_{n}=c, \quad \text { and } \quad m v_{x}=\sigma .
$$

3.2. Proprietary platform. The timing of the game is the following: (i) the platform provider chooses $x, p^{u}$, and $p^{d}$; (ii) users and developers decide whether to join the platform; and (iii) developers choose $\rho(j)$, and users choose how many applications to buy. The equilibrium concept is subgame perfect equilibrium. 
Since developers cannot invest in platform quality, equations (1) and (2) become

$$
\begin{aligned}
u(i) & =v(n, x)-\int_{0}^{n} \rho(j) d j-h(i)-p^{u}, \\
\pi(j) & =\rho(j) m-c(j)-p^{d} .
\end{aligned}
$$

In the third stage, developers choose the price of applications. Let $\rho^{*}(j)$ be the third-stage equilibrium price of application $j$. Price is determined differently when applications are substitutes and complements.

When applications are substitutes, the largest price an application developer may charge is $v_{n}$ (if the price of any application was greater than the marginal value of the last application, users would be better off not consuming that application), which means that application prices are $\rho^{*}(j)=v_{n}$ for all $j$.

When applications are complements, the equilibrium price is no longer $v_{n}$. To see this, note that if price was $v_{n}$, the total cost of a bundle of $n$ applications would be larger than its gross utility to users $\left(n v_{n}>v(n, x)-v(0, x)\right)$, and thus users would be better off not buying any application. In equilibrium, we must have $\int_{0}^{n} \rho(j) d j \leq v(n, x)-v(0, x)$. As long as $\int_{0}^{n} \rho(j) d j<v(n, x)-v(0, x)$, a developer may increase the price of its application without affecting user demand. Thus, in equilibrium $\int_{0}^{n} \rho(j) d j=v(n, x)-v(0, x)$. Let $w(n, x)=\frac{v(n, x)-v(0, x)}{n}$ be the average contribution of applications to consumer utility, and note that $w(n, x)$ is increasing in $n$ and $x$. In a symmetric equilibrium, all developers charge the same price, and application prices are $\rho^{*}(j)=w$ for all $j$.

In the second stage, users and developers choose whether to access the platform. The marginal entrants, $m$ and $n$, satisfy $v(n, x)-n \rho^{*}=h(m)+p^{u}$, and $m \rho^{*}=$ $c(n)+p^{d}$. From here, we obtain the inverse demand functions:

$$
\begin{aligned}
p^{u} & =v(n, x)-n \rho^{*}-h(m), \\
p^{d} & =m \rho^{*}-c(n) .
\end{aligned}
$$

Since $\rho^{*}$ does not depend on $m, \partial p^{u} / \partial m=-h_{m}<0$ for substitutes and complements. With substitutes, $\partial p^{d} / \partial n=m v_{n n}-c_{n}$, which is always negative. With complements, $\partial p^{d} / \partial n=m w_{n}-c_{n}$, which is negative only if $n c_{n}>m\left(v_{n}-\right.$ $w)$. We assume this condition holds.

In the first stage, the platform provider chooses $x, p^{u}$, and $p^{d}$ to maximize profits $m p^{u}+n p^{d}-\sigma x$. The following proposition characterizes the equilibrium. 
Proposition 1 (Proprietary platform). An equilibrium exists and is unique. The measure of users and developers and the investment in platform quality $(m, n, x)$ satisfy $v=h+m h_{m}, m v_{n}=c+n c_{n}$, and $m v_{x}=\sigma$. If applications are substitutes, $\rho^{*}=v_{n}, p^{u}=m h_{m}-n v_{n}$, and $p^{d}=n c_{n}$. If applications are complements, $\rho^{*}=w, p^{u}=m h_{m}-n w$, and $p^{d}=n c_{n}-m\left(v_{n}-w\right)$.

The marginal user and developer obtain zero utility and profit in equilibrium. Therefore, the net utility of user $i<m$ in equilibrium is $u(i)=h(m)-h(i)$, and the profit of developer $j<n$ is $\pi(j)=c(n)-c(j)$.

The condition determining $x$ in the proprietary platform is the same as that of the first best. Therefore, if $m$ and $n$ were set at their socially optimal levels, investment would be optimal. A proprietary platform sets access prices in order to capture the full increase in user surplus due to an increase in $x$, and thus has strong incentives to invest in product quality.

However, the conditions determining $m$ and $n$ are different from those of the first best, which means that $x$ will be set at an inefficient level. Efficiency requires that the value of the platform is equal to the entry cost of the marginal user $(v=h)$, and that the marginal benefit of the marginal application is equal to the entry cost of the marginal developer, $\left(m v_{n}=c\right)$. The platform provider does not fully internalize the marginal benefits of increases in $m$ and $n$, and thus sets prices that lead to insufficient entry.

Turning to the analysis of prices, we see that the platform provider may choose to subsidize users when the price of applications is high, but that she will never subsidize developers (recall that $n c_{n}>m\left(v_{n}-w\right)$ when applications are complements).

3.3. Open platform. We now turn to the analysis of the open platform. By their very nature, open platforms have unstructured entry and investment. Therefore, $m, n$, and $x(j)$ are determined simultaneously in the first stage. Application prices, $\rho(j)$, are set in a second stage. Since access to the platform is free, equations (1) and (2) become:

$$
\begin{aligned}
u(i) & =v(n, x)-\int_{0}^{n} \rho(j) d j-h(i), \\
\pi(j) & =\rho(j) m-c(j)-\sigma x(j),
\end{aligned}
$$

where $x=\int_{0}^{n} x(j) d j$. 
In open platforms, payments between users and developers affect the incentives to join the platform and to invest in platform quality. Because application prices are determined differently when they are substitutes and complements, we study both cases separately. Proposition 2 summarizes the equilibrium choices of users and developers when applications are substitutes.

Proposition 2 (Open platform with substitute applications). An equilibrium exists. The measure of users and developers and the investment in platform quality $(m, n, x)$ satisfy $h=v-n v_{n}, c=m v_{n}$, and $m v_{n x}=\sigma$. Application prices are $\rho^{*}=v_{n}$.

In the case of open platforms, there may exist multiple equilibria. We will focus our analysis on the equilibrium with higher platform investment.

In equilibrium, users obtain $u(i)=h(m)-h(i)$, and developers earn $\pi(j)=$ $c(n)-c(j)-\sigma x(j)$. Because $h_{i}>0$ and $c_{j}>0$, larger equilibrium entry by users or developers implies more user utility and developer profit.

A given level of equilibrium aggregate investment, $x$, may result from different distributions of individual developer investments, $x(j)$. As long as $m v_{n x}>\sigma$, any developer will find it optimal to increase its investment in platform quality. In equilibrium, $m v_{n x}=\sigma$ regardless of who is investing.7 Note that since investment is open, developers may choose not to invest in platform quality. If $m v_{n}>c(j)$, developer $j$ will find it optimal to enter the platform. Thus, there will be entry until $m v_{n}=c$, and the marginal developer will not invest in platform quality.

The condition determining developer access is the same as that of the first best. However, the equilibrium is inefficient for two reasons. First, user access

${ }^{7}$ Our model can be thought as an approximation to a more general model with heterogeneous investment costs and small private benefits of investment for developers. Specifically, consider an open platform with substitute applications and suppose the indirect utility for user $i$ is

$$
u(i)=v(n, x)+\alpha \int_{0}^{n} z(x(j)) d j-\int_{0}^{n} \rho(j) d j-h(i),
$$

where $\alpha z(x(j))$ is the standalone contribution to utility of application $j$, with $\alpha \geq 0, z_{x}>0$, and $z_{x x}<0$. Finally, suppose that the investment cost of developer $j$ is $\sigma(j)$ and that $\sigma_{j}>0$. Application prices are $v_{n}+\alpha z_{x}(x(j))$, which means that $\alpha z_{x}(x(j))$ represents the private benefit of investment for developer $j$ (Llanes and de Elejalde, 2013). Optimal investments solve $m v_{n}=\sigma(j)-\alpha m z_{x}(x(j))$. Integrating across developers we obtain that the equilibrium investment solves

$$
m v_{n x}=\frac{1}{n} \int_{o}^{n} \sigma(j) d j+\alpha \frac{1}{n} \int_{o}^{n} m z_{x}(x(j)) d j
$$

which means that $m v_{n x}$ is close to the average of $\sigma(j)$ when $\alpha$ is close to zero. 
is suboptimal because applications are priced above their marginal cost. The total mark-up paid by users is $n v_{n}$. Second, developer incentives to invest are not socially optimal because developers do not fully internalize the effect of an increase in $x$ on user utility.

We turn now to the case of complement applications. Proposition 3 summarizes the equilibrium choices of users and developers.

Proposition 3 (Open platform with complement applications). An equilibrium exists. The measure of users and developers and the investment in platform quality $(m, n, x)$ satisfy $h=v-n w, c=m w$, and $m w_{x}=\sigma$. Application prices are $\rho^{*}=w$.

There are two important differences between the substitutes and complements cases. First, when applications are complements, developers extract all the surplus from users, and user utility ends up being $v(0, x)$. However, users may still benefit indirectly from having more developers, because it may lead to higher investment in the platform. Second, in the complements case, developer entry would be inefficient even if $m$ and $x$ were set at their optimal levels. The reason is that developer revenue depends on the average contribution of the application to consumer utility, instead of its marginal contribution, which is required for efficiency.

3.4. Comparison. In this section, we compare the equilibrium conditions for the monopoly proprietary and open platforms. First, we compare entry and investment incentives examining one condition at a time, holding everything else constant. Then, we present conditions under which an open platform may lead to higher investment than a proprietary platform. $]^{8}$ Figure 1 presents a summary of our results.

We begin by comparing the incentives for user access. In a proprietary platform, the platform provider internalizes the effect of monetary payments from users to developers, and chooses access prices to neutralize it. Thus, user access depends only on the extensive margin of demand. In an open platform, users do not have to pay an access price but they have to pay application prices, which depend on

\footnotetext{
${ }^{8}$ This paper focuses on comparing investment incentives for open and proprietary two-sided platforms. The model is very general, which allows us to derive general results on incentives to invest. Unfortunately, however, we cannot make precise comparisons of welfare and profits without assuming specific functional forms.
} 


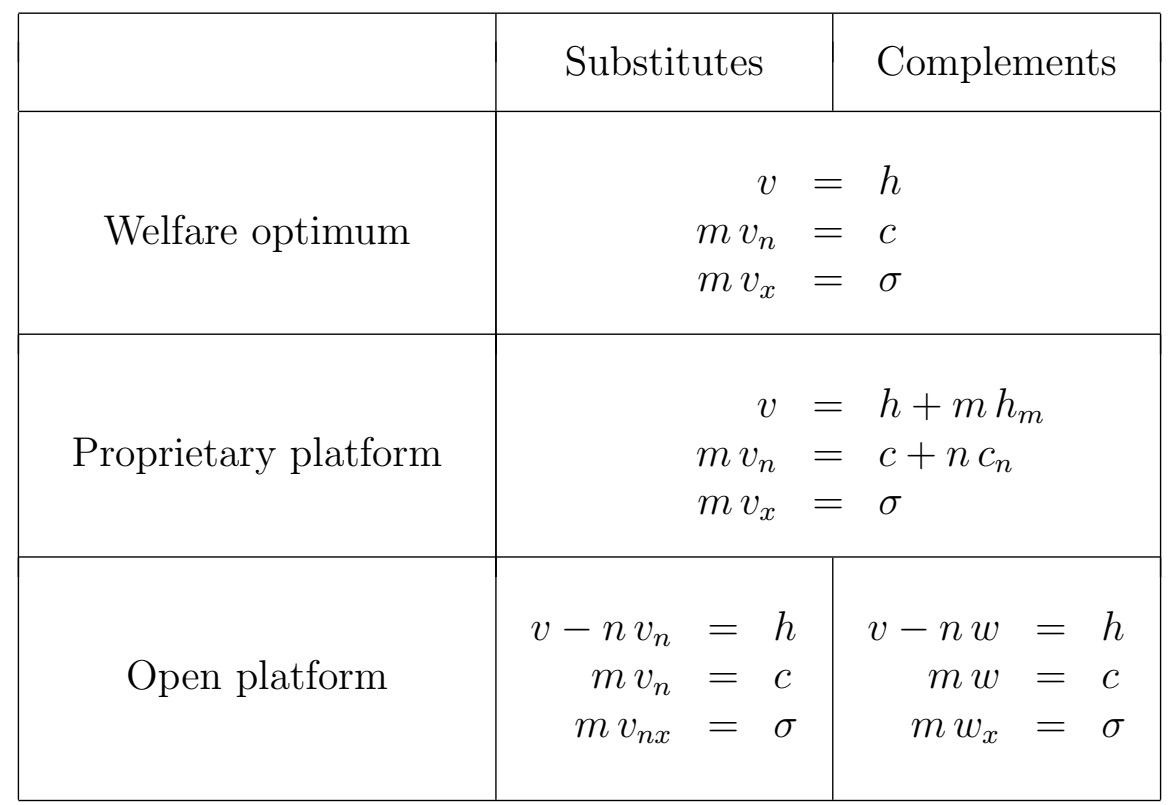

Figure 1. Equilibrium conditions for monopoly platforms. Functions $v(n, x)$ and $w(n, x)$ and their derivatives are evaluated at the equilibrium values of $m, n$, and $x$. Functions $h(i)$ and $c(j)$ and their derivatives are evaluated at the marginal user $i=m$ and developer $j=n$.

the marginal or average contribution of an additional application to user utility. Thus, user access depends only on the intensive margin of demand.

Therefore, the comparison of user access in proprietary and open platforms depends on how restrictive the extensive and intensive margins are; i.e., on the comparison between $m h_{m}$ and $n v_{n}$ in the substitutes case, and between $m h_{m}$ and $n w$ in the complements case.

Recall that the equilibrium access price for users is $p^{u}=m h_{m}-n v_{n}$ for substitutes and $p^{u}=m h_{m}-n w$ for complements. If intensive margin has a large impact on the demand for platform access, the proprietary platform may end up subsidizing users, in which case it will provide better incentives for user adoption.

The comparison of the incentives for developer access yields a different result: open platforms always provide stronger incentives for developer access for a given number of users and investment level. Open platforms provide good incentives for developer entry. Proprietary platforms restrict developer access because they always set a positive access price for developers. 
Finally, we compare equilibrium investment. The condition that determines quality investment in open platforms is $m v_{n x}=\sigma$ (substitutes) or $m w_{x}=\sigma$ (complements). For proprietary platforms, the condition is $m v_{x}=\sigma$. Holding everything else constant (i.e., taking $m$ and $n$ as given), equilibrium investment in an open platform is lower than in a proprietary platform. In the case of complements, this follows from $w_{x}<v_{x}$, which always holds. In the case of substitutes, even though $v_{n x}$ could be larger than $v_{x}$ from a mathematical point of view, it is only reasonable to assume that $v_{n x}<v_{x}$. To understand why, note that if the model had a discrete number of developers, $v_{n x}$ would be defined as $v_{x}(n, x)-v_{x}(n-1, x)$, which is always smaller than $v_{x}(n, x)$.

In any case, investment may be larger in an open platform compared to a proprietary one, since $m, n$, and $x$ are determined jointly. In particular, an open platform may lead to a larger number of users, which improves investment incentives. Also, an open platform may have higher investment if it leads to fewer developers. The reason is that when $v_{n n x}<0$, a decrease in the number of developers lowers competitive pressure and increases their incentives to invest in platform quality. Likewise an open platform may have higher investment if it leads to more developers and $v_{n n x}>0$. Proposition 4 summarizes these findings. In Section 3.5, we illustrate this result with the help of an example.

Proposition 4. A monopoly open platform may lead to higher investment than a monopoly proprietary platform only if one of the following conditions is met:

(i) The open platform leads to more users,

(ii) The open platform leads to more developers when investment and applications are complements with respect to developer revenues $\left(v_{n n x}>0\right)$, or

(iii) The open platform leads to fewer developers when investment and applications are substitutes with respect to developer revenues $\left(v_{n n x}<0\right)$.

3.5. Constant elasticity example. The following example illustrates that investment in platform quality may be larger when a platform is open. Let $v(x, n)=$ $x^{a} n^{b}$, where $0<a<1$ and $0<b<1$ are the elasticities of gross value with respect to investment and the number of applications. The assumption $b<1$ implies that applications are substitutes. We also assume that $2 a+b<1$, which guarantees that the second-order conditions for profit maximization are satisfied. Investment 
in platform quality and the measure of applications are complements, $v_{n x}>0$. Finally, let $h(i)=i, c(j)=j$, and $\sigma=1$.

Using the equations in Figure 1, we derive equilibrium adoption and investment. The social planner's solution is

$$
m^{s}=\left(a^{a} b^{-\frac{b}{2}}\right)^{\frac{1}{1-2 a-b}}, \quad n^{s}=\left(a^{a} b^{\frac{2 a-1}{2}}\right)^{\frac{1}{1-2 a-b}}, \quad x^{s}=\left(a^{1-b} b^{b}\right)^{\frac{1}{1-2 a-b}} .
$$

For the proprietary platform, the equations are

$$
m^{p}=2^{-\frac{1-a}{1-2 a-b}} m^{s}, \quad n^{p}=2^{-\frac{1-a}{1-2 a-b}} n^{s}, \quad x^{p}=2^{-\frac{1-a}{1-2 a-b}} x^{s} .
$$

Finally, for the open platform, we have

$$
\begin{gathered}
m^{o}=\left(a^{a} b^{\frac{a+b}{2}}(1-b)^{\frac{a+b-2}{2}}\right)^{\frac{1}{1-a-b}}, \quad n^{o}=\left(a^{a}(b(1-b))^{\frac{1}{2}}\right)^{\frac{1}{1-a-b}}, \\
x^{o}=\left(a^{1-b}(b(1-b))^{\frac{1}{2}}\right)^{\frac{1}{1-a-b}} .
\end{gathered}
$$

Due to the non-linearity of the equilibrium equations, it is not possible to find an explicit solution for the parameter values that lead to $x^{o}>x^{p}$. Figure 2 compares the first best investment level $\left(x^{*}\right)$, the investment in the open platform $\left(x^{o}\right)$, and the investment in the proprietary platform $\left(x^{p}\right)$ for different values of $b$ and $a=0.03$. As can be seen, the first best investment is higher than the investment in the open platform and the proprietary platform. The proprietary platform leads to higher investment when $b$ is small, and the open platform leads to higher investment when $b$ is large.

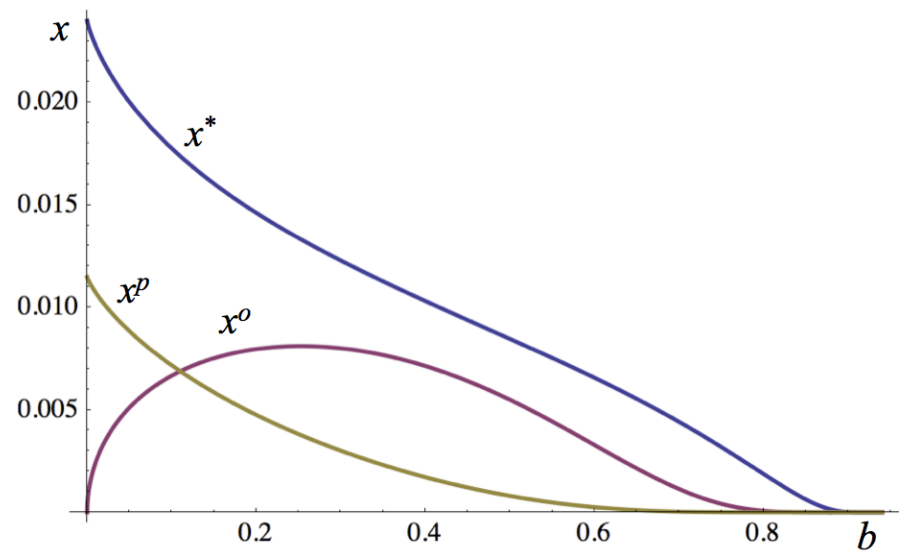

Figure 2. Comparison of equilibrium investments 


\section{Open ACCESS With CLOSED INVESTMENT}

In this section, we study a hybrid model of a proprietary platform that grants open access to developers, but still sets a positive access price to users and invests in platform quality (one-sided open-access platform). The model is the same as the one of Section 3.2 , but assuming that $p^{d}=0$. The platform provider may be constrained to offer free access to one side because of regulations or habits, or it may be too costly to observe access for one side. Proposition 5 shows the equilibrium of this game.

Proposition 5 (Open access with closed investment). When applications are substitutes, the measure of users and developers and the investment in platform quality $(m, n, x)$ satisfy $v=h+m h_{m}+\frac{n c_{n}}{c_{n}-m v_{n}} v_{n}, m v_{n}=c$, and $m v_{x}-$ $\frac{n c_{n}}{c_{n}-m v_{n n}} m v_{n x}=\sigma$. Equilibrium prices are $\rho^{*}=v_{n}$ and $p^{u}=m h_{m}+\frac{n v_{n}}{c_{n}-m v_{n n}} m v_{n n}$. When applications are complements, the measure of users and developers and the investment in platform quality $(m, n, x)$ satisfy $v=h+m h_{m}+\frac{n c_{n}-m\left(v_{n}-w\right)}{c_{n}-m w_{n}} w$, $m w=c$, and $m\left(v_{x}-n w_{x}\right)=\sigma$. Equilibrium prices are $\rho^{*}=w$ and $p^{u}=m h_{m}$.

A comparison of Propositions 1 and 5 shows that access and investment strategies have different effects on the equilibrium, but also have important interactions.

In a proprietary platform with closed access, investment incentives are strong because the platform provider can internalize indirect network effects between the two groups, and adjust access prices accordingly. Thus, the platform provider appropriates the contribution of investment to users' gross utility, $v_{x}$, which means that investment would be socially optimal if $m$ and $n$ were set at their first-best levels. With open access, the platform provider cannot internalize network effects, and thereby benefits only partially from her investment in platform quality. Thus, investment incentives would be suboptimal even if $m$ and $n$ were set at their firstbest levels.

As for access incentives, giving open access to developers improves their direct incentives to join the platform, but has a direct negative effect on user access and investment. Given that developers' revenues depend on user demand, which in turn depends on the investment in the platform, a one-sided open-access policy may reduce the number of developers in equilibrium. 


\section{Mixed Duopoly}

In this section, we extend the model to analyze competition between a proprietary platform and an open platform. For concreteness, we will focus on the case of substitute applications, but similar results hold for the case of complements.

We model the mixed duopoly as follows. There is one unit mass of singlehoming users, indexed by $i \in[0,1]$. User $i$ 's utility of consuming $n$ applications in the proprietary and open platforms are

$$
\begin{aligned}
& u^{p}(i)=v\left(n^{p}, x^{p}\right)-\int_{0}^{n^{p}} \rho^{p}(j) d j-p^{u}-h^{p}(i), \\
& u^{o}(i)=v\left(n^{o}, x^{o}\right)-\int_{0}^{n^{o}} \rho^{o}(j) d j-h^{o}(i),
\end{aligned}
$$

where superscripts $p$ and $o$ indicate whether the variable or function refers to the proprietary platform or to the open platform.

Access to the open platform is free. To guarantee that the market is covered, we assume that $\min _{i} h^{p}(i)$ and $\min _{i} h^{o}(i)$ are sufficiently low. The optimal choice of platform by users depends on $h(i)=h^{p}(i)-h^{o}(i)$, which measures the difference in the cost of learning how to use the proprietary vs. the open platform. Assume $h_{i}>$ 0 , with $\lim _{i \rightarrow 0} h(i)=-\infty$ and $\lim _{i \rightarrow 1} h(i)=\infty$. Let $m$ indicate the indifferent user. Then, $m$ is the measure of users choosing the proprietary platform, and $1-m$ is the measure of users choosing the open platform.

Developers multi-home. Thus we assume that it is inexpensive to adapt applications to run on both platforms. Even though the measure of applications is the same for both platforms, equilibrium application prices may differ across platforms because they depend on platform quality investments.

The timing is as follows: (i) the proprietary platform chooses $p^{u}, p^{d}$, and $x^{p}$; (ii) users choose which platform to join, and developers decide whether to develop an application and choose $x^{o}(j)$; and (iii) users and developers bargain over application prices $\rho^{p}(j)$ and $\rho^{o}(j)$. The timing reflects the fact that proprietary platforms are developed before they become accessible to users and developers, but that adoption and development are contemporaneous in open platforms. The equilibrium concept is subgame perfection.

In the third stage, users and developers bargain over application prices. The price of applications running on the proprietary platform is $v_{n}^{p}$, and the price of applications running on the open platform is $v_{n}^{o}$. 
In the second stage, the marginal user and developer satisfy $h(m)=v^{p}-v^{o}-$ $n\left(v_{n}^{p}-v_{n}^{o}\right)-p^{u}$ and $c(n)=m v_{n}^{p}+(1-m) v_{n}^{o}-p^{d}$. The inverse demands are

$$
\begin{aligned}
& p^{u}=v^{p}-v^{o}-n\left(v_{n}^{p}-v_{n}^{o}\right)-h, \\
& p^{d}=m v_{n}^{p}+(1-m) v_{n}^{o}-c,
\end{aligned}
$$

and the optimal investment in the open platform by developers is

$$
(1-m) v_{n x}^{o}=\sigma \text {. }
$$

In the first stage, the platform provider chooses $p^{u}, p^{d}$, and $x^{p}$ to maximize profits, taking into account that the second-stage equilibrium levels of $m, n$, and $x^{o}$ are functions of $p^{u}, p^{d}$, and $x^{p}$. Proposition 6 characterizes the equilibrium.

Proposition 6 (Mixed duopoly). An equilibrium exists and is unique. The measure of users and developers and investments in platform quality $\left(m, n, x^{o}, x^{p}\right)$ satisfy

$$
\begin{aligned}
h+m h_{m} & =\left(v^{p}-v^{o}\right)+\left(n v_{n x}^{o}-m v_{x}^{o}\right) \frac{v_{n x}^{o}}{(1-m) v_{n x x}^{o}}, \\
c+n c_{n} & =m\left(v_{n}^{p}-v_{n}^{o}\right)+\left(v_{n}^{o}+n v_{n n}^{o}\right)-\left(n v_{n x}^{o}-m v_{x}^{o}\right)\left(-\frac{v_{n n x}^{o}}{v_{n x x}^{o}}\right),
\end{aligned}
$$

$(1-m) v_{n x}^{o}=\sigma$, and $m v_{x}^{p}=\sigma$. Equilibrium access prices are

$$
\begin{aligned}
& p^{u}=m h_{m}-n\left(v_{n}^{p}-v_{n}^{o}\right)-\left(n v_{n x}^{o}-m v_{x}^{o}\right) \frac{v_{n x}^{o}}{(1-m) v_{n x x}^{o}}, \\
& p^{d}=n\left(c_{n}-v_{n n}^{o}\right)-\left(n v_{n x}^{o}-m v_{x}^{o}\right)\left(-\frac{v_{n n x}^{o}}{v_{n x x}^{o}}\right) .
\end{aligned}
$$

Proposition 6 shows that access prices are affected by investment incentives in the open platform. The proprietary-platform provider takes into account that her decisions affect the incentives to invest in the open platform, which in turn, affect platform membership decisions, and adjusts access prices accordingly.

To understand this result, consider the equilibrium prices $p^{u}$ and $p^{d}$, and assume that $x^{o}$ is fixed $\left(v_{n x}^{o}=0\right)$. In this case, the price equations are

$$
\begin{aligned}
p^{u} & =m h_{m}-n\left(v_{n}^{p}-v_{n}^{o}\right), \\
p^{d} & =n\left(c_{n}-v_{n n}^{o}\right) .
\end{aligned}
$$


Allowing for changes in $x^{o}$, we have

$$
\begin{aligned}
& p^{u}=m h_{m}-n\left(v_{n}^{p}-v_{n}^{o}\right)-\left(n v_{n x}^{o}-m v_{x}^{o}\right) \frac{v_{n x}^{o}}{(1-m) v_{n x x}^{o}}, \\
& p^{d}=n\left(c_{n}-v_{n n}^{o}\right)-\left(n v_{n x}^{o}-m v_{x}^{o}\right)\left(-\frac{v_{n n x}^{o}}{v_{n x x}^{o}}\right) .
\end{aligned}
$$

The new terms in the price equations measure the indirect effect of changes in $m$ and $n$ on profits as they operate through $x^{o}$. To see this, note that the effect of a change in $x^{o}$ on the revenues of the proprietary firm is

$$
\begin{aligned}
m \frac{\partial p^{u}}{\partial x^{o}}+n \frac{\partial p^{d}}{\partial x^{o}}= & m\left(-v_{x}^{o}+n v_{n x}^{o}\right)+n(1-m) v_{n x}^{o} \\
& n v_{n x}^{o}-m v_{x}^{o},
\end{aligned}
$$

and the effects of changes in $m$ and $n$ on $x^{o}$ are

$$
\begin{aligned}
\frac{\partial x^{o}}{\partial m} & =\frac{v_{n x}^{o}}{(1-m) v_{n x x}^{o}}, \\
\frac{\partial x^{o}}{\partial n} & =-\frac{v_{n n x}^{o}}{v_{n x x}^{o}} .
\end{aligned}
$$

Thus, the effect of the open platform's investment in the structure of access prices depends on: (i) how an increase in $m$ and $n$ affect the incentives to invest in the open platform, and (ii) how an increase in the investment in the open platform affects the revenues of the proprietary platform.

An increase in the investment in the open platform has two opposing effects on the revenues of the proprietary platform. On one hand, an increase in $x^{o}$ raises the gross value of the open platform, thereby lowering user-side revenues for the proprietary platform. On the other hand, an increase in $x^{o}$ raises application prices and developer revenues, leading to higher developer-side revenues.

Therefore, an increase in the quality of the open platform may lead to higher revenues for the proprietary platform. This seemingly counter-intuitive result is due to multi-homing. The proprietary-platform provider gains more on the developer side when there is more developer access, and access is partly determined by developers' revenues in the open platform.

Turning to the analysis of the effects of changes in $m$ and $n$ on $x^{o}$, an increase in $m$ decreases the market share of the open platform, and therefore lowers the incentives to invest in it. On the other hand, an increase in $n$ may lead to higher or lower investment in the open platform, depending on the sign of $v_{n n x}^{o}$, which 
is positive (negative) when $n$ and $x$ are complements (substitutes) with respect to application price $\rho^{o}=v_{n}^{o}$. If $v_{n n x}^{o}>0$, an increase in $n$ leads to a higher $v_{n x}^{o}$, and thus leads to more incentives to invest. Likewise, if $v_{n n x}^{o}<0$, an increase in $n$ lowers incentives to invest.

Depending on the sign of these effects, $p^{u}$ and $p^{d}$ may increase or decrease relative to a situation without investment. For example, if an increase in $x^{o}$ lowers the revenues of the proprietary platform, and an increase in $n$ leads to higher investment in the open platform, the proprietary platform should lower $p^{u}$ and raise $p^{d}$, in comparison to the model without investment. Figure 3 summarizes the strategic implications of investment incentives on access prices.

\begin{tabular}{c|c|c|c|}
\multicolumn{1}{c}{} & \multicolumn{2}{c}{$\begin{array}{r}\text { Effect of open-platform investment on } \\
\text { proprietary-platform revenues }\end{array}$} \\
\cline { 2 - 4 } & & Negative & Positive \\
\cline { 2 - 4 } $\begin{array}{c}\text { Effect of developer } \\
\text { access on open- } \\
\text { platform investment }\end{array}$ & Negative & $\begin{array}{c}\text { Lower user access prices } \\
\text { Raise developer access prices }\end{array}$ & $\begin{array}{c}\text { Raise user access prices } \\
\text { Raise developer access prices }\end{array}$ \\
\cline { 2 - 4 } & Positive & $\begin{array}{c}\text { Lower user access prices } \\
\text { Lower developer access prices }\end{array}$ & $\begin{array}{c}\text { Raise user access prices } \\
\text { Lower developer access prices }\end{array}$ \\
\cline { 2 - 4 } & &
\end{tabular}

FiguRE 3. Effect of investment incentives on access prices in the duopoly

Finally, from the equations determining platform investments, we obtain the following ratio in equilibrium:

$$
\frac{1-m}{m}=\frac{v_{n}^{p}}{v_{n x}^{o}}
$$

Therefore, equilibrium investment in the proprietary platform increases relative to the investment in the open platform as the equilibrium market share of the proprietary platform increases.

In summary, we find that the effect of investment in the open platform on the structure of access prices depends on: (i) how changes in the number of developers affect investments in the open platform, and (ii) how investments in the open platform affect the revenues of the proprietary platform. 
We also show that when developers multi-home, the proprietary platform may benefit from higher quality investment in the open platform, which explains why proprietary firms may choose to contribute to the development of competing opensource platforms.

\section{Conclusion}

We have examined models of open-source and proprietary two-sided platforms to study equilibrium investment in quality. Our analysis has provided answers to three important questions that had not been tackled before in the literature: (i) How are the incentives to invest in platform quality affected by the degree of platform openness? (ii) What is the relation between access and investment strategies? and (iii) How are access prices and incentives to invest in platform quality moderated by competition between proprietary and open two-sided platforms?

Regarding the first question, we find that investment incentives are stronger in a proprietary platform for a given level of user and developer access, but that an open platform may still lead to higher investment if one of the following conditions is met: (i) the open platform attracts more users, or (ii) the open platform attracts fewer developers and the effect of a change in the number of applications on developer revenue decreases with investment in platform quality, or (iii) the open platform attracts more developers and the effect of a change in the number of applications on developer revenue increases with investment in platform quality. Therefore, we find that the successful development of an open platform may require limited developer access.

For the second question, we find that investment incentives depend on the access policies in place. For example, opening one side of a proprietary platform may lead to fewer users and developers and to lower investment, compared to a proprietary platform with closed access on both sides. If the proprietary platform cannot price both sides, it cannot internalize indirect network effects and thus, has weaker investment incentives. Lower investment, in turn, may lead to less adoption by users and developers.

Finally, for the question of incentives to invest and competition, we find that investment incentives in the open platform affect access prices and investment incentives in the proprietary platform. In particular, the structure of access prices of 
the proprietary platform depends on: (i) how changes in the number of developers affect the incentives to invest in the open platform, and (ii) how investment in the open platform affects the revenues of the proprietary platform. Also, we find that a proprietary platform may benefit from higher investment in the open platform. The reason is that when developers multi-home, the proprietary-platform provider gains more when more applications are developed, and the number of applications is partly determined by developers' revenues in the open platform.

We hope to have provided a solid first step to better understand incentives to invest in proprietary and open platforms. Our analysis has important managerial implications. First, we show under which conditions open source may lead to high investment in platform quality, which has important implications for profitmaximizing and non-profit firms participating on operating-system or applications markets. Second, we show that access and investment policies have different effects on the equilibrium, but also have important interactions. For example, introducing an open-access policy in an otherwise proprietary platform may improve access incentives at the cost of lowering investment incentives. Third, we show how access prices should be set when a proprietary platform competes directly with an open-source platform. Finally, we show that open platforms may be complementary to proprietary platforms, especially when the proprietary platform needs to construct an installed base of applications.

In order to focus our analysis on the incentives for platform investment, we have made several simplifying assumptions, such as not considering direct network effects among users, restricting heterogeneity to adoption costs for users and development costs for developers, and abstracting from design conflicts in open-source development. Extending the model to allow for direct network effects, heterogeneity in user valuations, and code forking present interesting directions for further research.

\section{Appendix: Proofs of Propositions in teXt}

Proof of Proposition 1. There is a unique pair of prices $p^{u}, p^{d}$ for each pair $m, n$, so finding the optimal $m$ and $n$ is equivalent to finding the optimal $p^{u}$ and

$p^{d}$. Replacing prices by inverse demand functions in the profit function we obtain $m\left(v-n \rho^{*}-h(m)\right)+n\left(m \rho^{*}-c(n)\right)-\sigma x$. Rearranging terms, profits can be rewritten as $m v-m h(m)-n c(n)-\sigma x$. 
The first-order conditions with respect to $(m, n, x)$ are $v=h+m h_{m}, m v_{n}=$ $c+n c_{n}$ and $m v_{x}=\sigma$. Assuming $h_{m m}$ and $c_{n n}$ are positive, or negative but not too large in absolute value, the second-order conditions will hold, and there will be at least one local maximum. If there is more than one local maximum, the firm will choose the one with the largest profit (i.e., the global maximum).

Substituting the first two expressions in the inverse demand functions, we obtain the optimal access prices. There is a unique pair $\left(p^{u}, p^{d}\right)$ for a given triple $(m, n, x)$.

Finally, even though in the second stage users and developers may coordinate in different second-stage equilibria for a given pair of access prices (i.e., there may be more than one pair $m, n$ solving $v=h+m h_{m}$ and $m v_{n}=c+n c_{n}$ ), only one combination $m, n$ will be part of the Nash equilibrium of the complete game (the one corresponding to the optimal prices $p^{u}, p^{d}$ ), which is a condition for subgame perfect equilibrium. Thus, the equilibrium is unique.

Proof of Proposition 2. By the arguments brought forward in Section 3.2, application price is $v_{n}$. In the first stage, users and developers choose whether to enter the platform, and developers choose how much to invest in platform quality. In choosing how much to invest, developers solve

$$
\max _{x(j)} m v_{n}(n, x)-c(j)-\sigma x(j) .
$$

The first-order conditions yield $m v_{n x}=\sigma$. The marginal user and developer obtain zero utility and profit. The marginal agents do not invest in platform innovation. Therefore, in equilibrium we must have $v-n v_{n}-h(m)=0$, and $m v_{n}-c(n)=0$.

Proof of Proposition 3. The proof follows similar steps as the proof of Proposition 2, taking into account that the price of applications is now $w$.

Proof of Proposition 4. In a monopoly proprietary platform, equilibrium investment solves $m v_{x}=\sigma$. In a monopoly open platform, investment solves $m v_{n x}=\sigma$. As we explained above, for given $n, x$, it makes sense to assume that $v_{x}>v_{n x}$. Thus, if $m$ and $n$ are the same in both platforms, investment in the proprietary platform will be higher. Suppose now that $v_{n n x}<0$ (the proof for $v_{n n x}>0$ is analogous) and that in equilibrium $n$ is larger for the open platform. Then, it is clear that investment can be larger in the open platform only if it leads to a higher $m$. Suppose now that $m$ is smaller in the open platform. Then, 
$v_{n x}>v_{x}$ and investment is larger in the open platform only if it leads to fewer developers.

Proof of Proposition 5. When applications are substitutes, the measure of users and developers is determined by $x$ and $p^{u}$ through the equations $p^{u}=$ $v-n v_{n}-h$, and $0=m v_{n}-c$. Using the implicit function theorem, we obtain the following derivatives:

$$
\begin{aligned}
\frac{\partial m}{\partial p^{u}} & =\frac{m v_{n n}-c_{n}}{c_{n} h_{m}+\left(n v_{n}-m h_{m}\right) v_{n n}} \\
\frac{\partial n}{\partial p^{u}} & =\frac{-v_{n}}{c_{n} h_{m}+\left(n v_{n}-m h_{m}\right) v_{n n}} \\
\frac{\partial m}{\partial x} & =\frac{c_{n}\left(v_{x}-n v_{n x}\right)-m v_{n n} v_{x}}{c_{n} h_{m}+\left(n v_{n}-m h_{m}\right) v_{n n}} \\
\frac{\partial n}{\partial x} & =\frac{m v_{n x} h_{m}+\left(v_{x}-n v_{n x}\right) v_{n}}{c_{n} h_{m}+\left(n v_{n}-m h_{m}\right) v_{n n}}
\end{aligned}
$$

The platform provider chooses $p^{u}$ and $x$ in order to maximize $p^{u} m\left(p^{u}, x\right)-$ $\sigma x$. Introducing the derivatives obtained above in the first-order conditions, we obtain the results stated in the proposition. The proof for the complementary applications case is analogous.

Proof of Proposition 6 . Existence and uniqueness follow from similar arguments than those of Proposition 1. The first-order conditions are $m+p^{u} \frac{d m}{d p^{u}}+$ $p^{d} \frac{d n}{d p^{u}}=0, p^{u} \frac{d m}{d p^{d}}+n+p^{d} \frac{d n}{d p^{d}}=0$ and $p^{u} \frac{d m}{d x^{p}}+p^{d} \frac{d n}{d x^{p}}-\sigma=0$. The optimal choices depend on the derivatives of $m\left(p^{u}, p^{d}, x^{p}\right)$ and $n\left(p^{u}, p^{d}, x^{p}\right)$ with respect to $p^{u}, p^{d}$, and $x^{p}$. We will show how to obtain $\frac{d m}{d p^{u}}$ (the other derivatives are obtained similarly). The total differentials of equations (5), (6) and (7) with respect to $p^{u}$ are

$$
\begin{aligned}
1 & =-h_{m} \frac{d m}{d p^{u}}+\left(v_{n}^{p}-v_{n}^{o}-n\left(v_{n n}^{p}-v_{n n}^{o}\right)\right) \frac{d n}{d p^{u}}-\left(v_{x}^{o}+n v_{n x}^{o}\right) \frac{d x^{o}}{d p^{u}} \\
0 & =\left(v_{n}^{p}-v_{n}^{o}\right) \frac{d m}{d p^{u}}+\left(m v_{n n}^{p}+(1-m) v_{n n}^{o}-c_{n}\right) \frac{d n}{d p^{u}}+(1-m) v_{n x}^{o} \frac{d x^{o}}{d p^{u}} \\
0 & =-v_{n x}^{o} \frac{d m}{d p^{u}}+(1-m) v_{n n x}^{o} \frac{d n}{d p^{u}}+(1-m) v_{n x x}^{o} \frac{d x^{o}}{d p^{u}},
\end{aligned}
$$

which constitute a system of three equations with three unknowns. Solving for $d m / d p^{u}$, we obtain

$$
\frac{d m}{d p^{u}}=(1-m) v_{n x x}^{o}\left((1-m) v_{n n x}^{o} v_{n x}^{o}-\left(v_{n n}^{o}+m\left(v_{n n}^{p}-v_{n n}^{o}\right)-c_{n}\right) v_{n x x}^{o}\right) D^{-1},
$$


where

$$
\begin{aligned}
D= & (1-m)\left(\left(v_{n}^{p}-v_{n}^{o}\right) v_{n x x}^{o}+v_{n x}^{o} v_{n x}^{o}\right) \\
& \left(\left(v_{n}^{p}-v_{n}^{o}-n\left(v_{n n}^{p}-v_{n n}^{o}\right)\right) v_{n x x}^{o}+\left(v_{x}^{o}+n v_{n x}^{o}\right) v_{n n x}^{o}\right) \\
& +\left(h_{m}(1-m) v_{n x x}^{o}+\left(v_{x}^{o}+n v_{n x}^{o}\right) v_{n x}^{o}\right) \\
& \left(\left(m v_{n n}^{p}+(1-m) v_{n n}^{o}-c_{n}\right) v_{n x x}^{o}-(1-m) v_{n x}^{o} v_{n n x}^{o}\right)
\end{aligned}
$$

Introducing the derivatives in the first-order conditions for the proprietary platform and solving for $p^{u}, p^{d}$, and $x^{p}$, we obtain the results stated in the proposition.

\section{REFERENCES}

Armstrong, M. (2006): "Competition in two-sided markets," RAND Journal of Economics, 37(3), 668-691.

Belleflamme, P., And M. Peitz (2010): "Platform competition and seller investment incentives," European Economic Review, 54(8), 1059-1076.

Bitzer, J. (2004): "Commercial versus open source software: The role of product heterogeneity in competition," Economic Systems, 28(4), 369-381.

Caillaud, B., And B. Jullien (2003): "Chicken \& egg: Competition among intermediation service providers," RAND Journal of Economics, 34(2), 309328.

Casadesus-Masanell, R., and P. Ghemawat (2006): "Dynamic mixed duopoly: A model motivated by Linux vs. Windows," Management Science, $52(7), 1072-1084$.

Casadesus-Masanell, R., and G. Llanes (2011): "Mixed source," Management Science, 57(7), 1212-1230.

Casadesus-Masanell, R., and F. Ruiz-Aliseda (2008): "Platform competition, compatibility, and social efficiency," Working paper 08-32, NET Institute, New York.

Corbet, J., G. Kroah-Hartman, and A. McPherson (2012): Linux Kernel Development: How Fast it is Going, Who is Doing It, What They are Doing, and Who is Sponsoring It. The Linux Foundation, San Francisco, CA.

Economides, N., and E. Katsamakas (2006a): "Linux vs. Windows: A comparison of application and platform innovation incentives for open source and proprietary software platforms," in The Economics of Open Source Software 
Development, ed. by J. Bitzer, and P. J. H. Schröder, chap. 10. Elsevier B. V., Amsterdam, Netherlands.

(2006b): "Two-sided competition of proprietary vs. open source technology platforms and the implications for the software industry," Management Science, 52(7), 1057-1071.

Fershtman, C., And N. Gandal (2011): "A brief survey of the economics of open source software," in The New Palgrave Dictionary of Economics, ed. by S. N. Durlauf, and L. E. Blume. Palgrave Macmillan, Basingstoke, UK.

Gaudeul, A. (2005): "Competition between open-source and proprietary software: The (La)TeX case study," Discussion paper, University of East Anglia, Norwich, UK.

Hagiu, A. (2006a): "Pricing and commitment by two-sided platforms," RAND Journal of Economics, 37(3), 720-737. (2006b): "Proprietary vs. open two-sided platforms and social efficiency," Working paper 09-113, Harvard Business School, Boston.

(2007): "Merchant or two-sided platform?," Review of Network Econom$i c s, 6(2)$.

Lee, D., and H. Mendelson (2008): "Divide and conquer: Competing with free technology under network effects," Production and Operations Management, $17(1), 12-28$.

Lerner, J., And J. Tirole (2005): "The economics of technology sharing: Open source and beyond," Journal of Economic Perspectives, 19(2), 99-120.

Lin, M., S. Li, And A. Whinston (2011): "Innovation and price competition in a two-sided market," Journal of Management Information Systems, 28(2), 171-202.

Llanes, G., and R. De Elejalde (2013): "Industry equilibrium with opensource and proprietary firms," International Journal of Industrial Organization, 31(1), 36-49.

Mustonen, M. (2003): "Copyleft-the economics of Linux and other open source software," Information Economics and Policy, 15(1), 99-121.

Nocke, V., M. Peitz, and K. Stahl (2007): "Platform ownership," Journal of the European Economic Association, 5(6), 1130-1160.

Rochet, J.-C., And J. Tirole (2003): "Platform competition in two-sided markets," Journal of the European Economic Association, 1(4), 990-1029. 
(2006): "Two-sided markets: A progress report," RAND Journal of Economics, 37(3), 645-667.

Spulber, D. F. (1996): "Market making by price setting firms," Review of Economic Studies, 63, 559-580.

- (2006): "Firms and networks in two-sided markets," in Handbooks in Information Systems, ed. by E. Terrence Hendershott, pp. 137-200. Elsevier, Amsterdam.

von KRogh, G., And E. vON Hippel (2006): "The promise of research on open source software," Management Science, 52(7), 975-983.

WeyL, E. (2010): "A price theory of multi-sided platforms," American Economic Review, 100(4), 1642-1672.

ZHAO, L. (2010): "Quantity constraints on two-sided platforms: The role of quality uncertainty," Harvard College Senior Thesis. 